\title{
Prospects for supersymmetric charged Higgs boson discovery at the Fermilab Tevatron and the CERN Large Hadron Collider
}

\author{
Alexander Belyaev, ${ }^{1}$ David Garcia, ${ }^{2}$ Jaume Guasch, ${ }^{3}$ and Joan Solà ${ }^{4}$ \\ ${ }^{1}$ Physics Department, Florida State University, Tallahassee, Florida 32306-4350 \\ ${ }^{2}$ Theory Division, CERN, CH-1211 Geneva 23, Switzerland \\ ${ }^{3}$ Institut für Theoretische Physik, Universität Karlsruhe, Kaiserstraße 12, D-76128 Karlsruhe, Germany \\ ${ }^{4}$ Departament d'Estructura i Constituents de la Matèria and Institut de Física d'Altes Energies, Universitat de Barcelona, Diagonal 647, \\ E-08028, Barcelona, Catalonia, Spain
}

(Received 6 May 2001; published 7 January 2002)

\begin{abstract}
We investigate the prospects for heavy charged Higgs boson production through the mechanisms $p \bar{p}(p p)$ $\rightarrow H^{ \pm} t b+X$ at the upgraded Fermilab Tevatron and at the upcoming LHC at CERN, respectively. We focus on the minimal supersymmetric standard model case and include the leading quantum corrections at high $\tan \beta$. A detailed study is performed for all important production modes and basic background processes for the $t \bar{t} b \bar{b}$ signature. At the upgraded Tevatron a charged Higgs signal is potentially viable in the $220-250 \mathrm{GeV}$ range or excluded at $95 \%$ C.L. up to $300 \mathrm{GeV}$. At the LHC, a $H^{ \pm}$of mass up to $800 \mathrm{GeV}$ can be discovered at $5 \sigma$ or else be excluded up to a mass of $\sim 1.5 \mathrm{TeV}$. The presence of SUSY quantum effects may highly influence the discovery potential in both machines and can typically shift these limits by $200 \mathrm{GeV}$ at the LHC.
\end{abstract}

DOI: 10.1103/PhysRevD.65.031701

PACS number(s): 12.60.Jv, 12.60.Fr, 13.85.Ni, 14.80.Cp

Although the standard model (SM) is a highly successful theory its theoretical structure relies on the Higgs mechanism, which still lacks direct experimental verification. The last results from the CERN $e^{+} e^{-}$collider LEP, suggesting a light neutral Higgs boson of about $115 \mathrm{GeV}$ [1], are encouraging, but we will have to wait for the news from the upgraded Fermilab Tevatron or from the upcoming Large Hadron Collider at CERN to see if this result is either confirmed or dismissed. For intermediate masses above the LEP limit and below $180 \mathrm{GeV}$ there is a chance for the Tevatron, but for higher masses up to $1 \mathrm{TeV}$ one needs the LHC. However, even if a neutral Higgs boson is discovered, the principal question will stand immutable at the forefront of elementary particle research: is the minimal SM realized in nature or does a model beyond the SM exist with an extended Higgs sector? In most of these extensions [2], the physical spectrum contains neutral Higgs particles and some of them may mimic the SM one. But in general they also involve charged Higgs bosons $H^{ \pm}$, and this introduces an obvious distinctive feature. In this Rapid Communication we report on the main results from a dedicated study of the $H^{ \pm}$production in association with the top quark in hadron colliders. We restrict our analysis to the Higgs sector of the minimal supersymmetric standard model (MSSM) with special emphasis on the role played by the SUSY radiative corrections. This is a timely issue, given the fact that the Tevatron run II is presently running. Furthermore, the subject should also be of interest for the LHC, which is the main hadron collider around the corner.

The relevant mechanisms on which we will concentrate,

$$
p \bar{p}(p p) \rightarrow H^{ \pm} t b+X \quad(\text { Tevatron })(\text { LHC }),
$$

are long known to be the leading ones for $H^{ \pm}$production at high $\tan \beta[3]$. They constitute the charged counterpart of the process $p \bar{p}(p p) \rightarrow H t \bar{t}+X$ for associated Higgs boson pro- duction in the SM [4] - recently reexamined in [5] for the Tevatron-and of $p \bar{p}(p p) \rightarrow h t \bar{t}+X$ for neutral MSSM Higgs boson production $h=h^{0}, H^{0}, A^{0}$ [2] at the Tevatron and the LHC [6]. While the study of Eq. (1) has been further addressed in the literature [7], to the best of our knowledge all the works on this subject-except a first estimation in [8] - do stick to a tree-level computation without including MSSM quantum effects, in spite of the fact that some of them explicitly admit that the sort of charged Higgs boson they are dealing with is of the MSSM type. Therefore, they are unavoidably affected by some drawbacks. In the present work we have implemented several additional features which improve in a substantial manner our knowledge on the real capability for the mechanisms (1) to produce a charged Higgs boson or to put limits on its mass within the MSSM. First, we include the leading SUSY radiative corrections (both strong and electroweak) along with an analysis of the off-shell effects. Second, we perform a beyond-the-partonlevel simulation of events, which includes the toy-detector simulation, jet fragmentation, and initial and final radiation effects. Third, we include a proper kinematical analysis of the $g g \rightarrow H^{+} \bar{t} b$ and $g \bar{b} \rightarrow H^{+} \bar{t}$ subprocesses. While the standard recipe to remove the overlap between these two amplitudes is well known at the level of total cross-sections [10], the correct combination of the differential distributions, as it is presented here, is a novel feature that follows the method based on Ref. [11].

A realistic study of charged Higgs boson physics cannot be accomplished without including the information provided by radiative corrections. These are not only potentially large in the computation of the MSSM Higgs boson masses themselves but also in the interaction vertices and self-energies of given processes, particularly in the decay of the top quark into charged Higgs bosons and for the hadronic decays of the MSSM Higgs bosons [12,13]. 


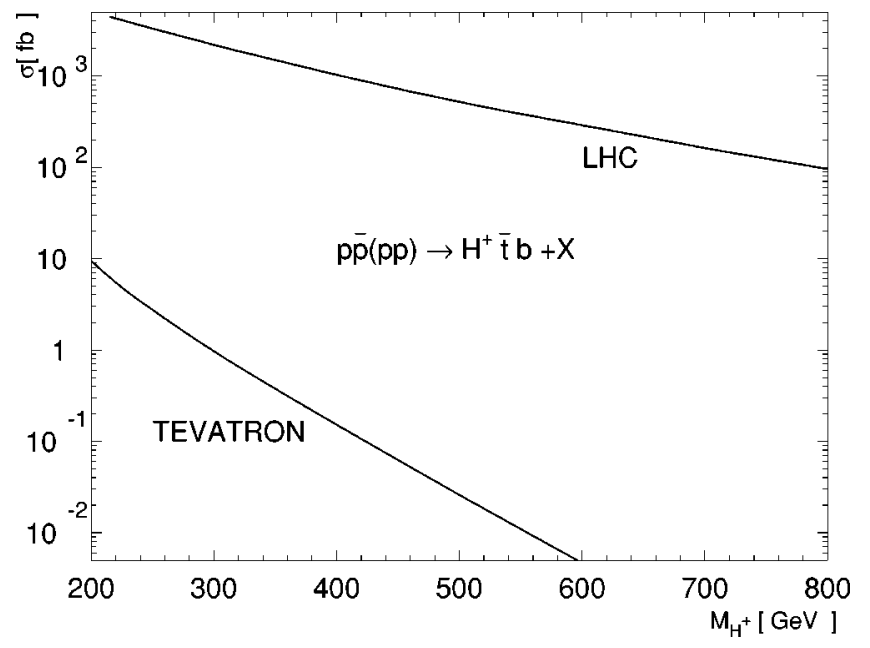

FIG. 1. Tree-level cross-sections (1) versus $M_{H^{ \pm}}$at the Tevatron run II ( $2 \mathrm{TeV})$ and $\mathrm{LHC}(14 \mathrm{TeV})$ for $\tan \beta=50$. We used the pole masses $m_{t}=175 \mathrm{GeV}, m_{b}=4.6 \mathrm{GeV}$.

Among the radiative corrections affecting process (1) we have the conventional QCD effects. They are unknown at present, and they are usually parametrized in terms of a $K$ factor [7]. We shall follow this practice here, but we wish to remark that two independent calculations on the crosssection for the companion SM process $p \bar{p}(p p) \rightarrow H t \bar{t}+X$ at the next-to-leading order (NLO) in QCD have appeared very recently in the literature [14]. The result is that at the Tevatron the NLO effects for SM Higgs production are negative, and can be approximately described by a $K$ factor ranging between $0.8-1$, whereas at the LHC they are positive and the $K$ factor lies between 1.2-1.4 depending on the scale. This is in agreement with early expectations within the effective Higgs approximation (EHA) [15]. As we have said, the corresponding calculation for the charged Higgs production process under study is not available, and moreover the EHA is not valid here because the bottom quark is lighter than the Higgs boson. Nevertheless, in contrast to the Ht $\bar{t}$ production case in the SM, we can foresee a large $K$ factor for process (1) at both the Tevatron and the LHC. This expectation is based on standard facts on heavy quark production at hadron colliders [16], in particular on bottom quark production, in combination with the known QCD results on neutral Higgs production in $e^{+} e^{-}$annihilation [17]. Indeed, while in the $\mathrm{SM}$ process $p \bar{p}(p p) \rightarrow H t \bar{t}+X$ the initial state is largely dominated by $q \bar{q}$ annihilation (due to the heavy top quarks in the final state) the situation in process (1) is very different. Here the presence of a much lighter quark in the final state (the bottom quark) causes the $g g$ fusion partonic mode, in combination with the $g \bar{b}$ initiated amplitude, to be of comparable importance (for the Tevatron) or even highly dominant (at the LHC). We have numerically checked these facts. As a consequence, large NLO effects are expected in the process (1) from general arguments on inclusive $b$ production [16]. Moreover, the renormalization of the Higgs Yukawa coupling $H^{ \pm} t b$ in the final state is connected to the renormalization of the quark masses. Therefore, as in $e^{+} e^{-}$

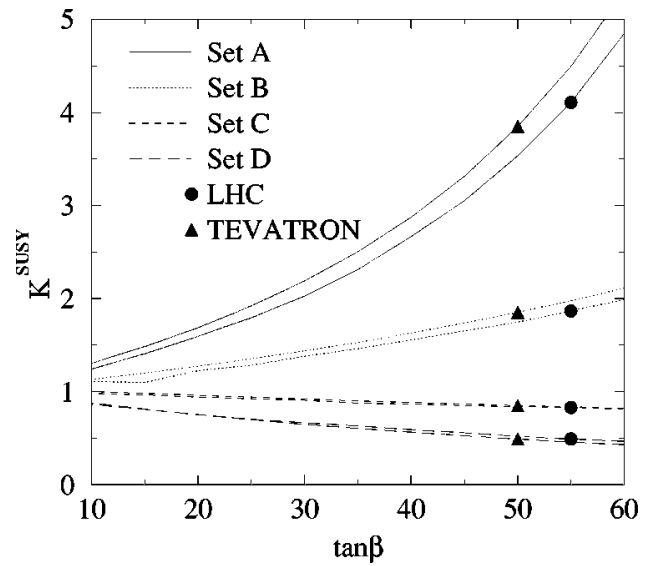

FIG. 2. $K_{\mathrm{SUSY}}=\sigma^{\mathrm{SUSY}} / \sigma^{\text {tree }}$ factor for the signal (1) as a function of $\tan \beta$ for the typical sets of parameters in Table I, at the Tevatron (for $M_{H^{ \pm}}=250 \mathrm{GeV}$ ) and the LHC (for $M_{H^{ \pm}}$ $=500 \mathrm{GeV})$.

$\rightarrow h \bar{b} b[17]$, one absorbs the large (negative) QCD effects of the final state into the running quark masses. After that the resummation of the collinear mass singularities in the initial state, potentially represented by large logarithms of the type $\ln \left(M_{H^{ \pm}} / m_{b}\right)$ - having no counterpart in the SM case-leads one to typically expect $K=1.5-2$ for the corresponding QCD corrections at the the NLO [18].

On the other hand, the supersymmetric quantum effects (from squarks, gluinos, and charginos) can also be very important for the processes (1). We have explicitly computed the leading effects, and included also the virtual contributions from the MSSM Higgs bosons. At very large values of $\tan \beta$, the SUSY radiative effects can play an important role in increasing the reach of the Tevatron and the LHC for the charged SUSY Higgs search. Furthermore, the SUSY effects are essential to identify the charged Higgs boson as potentially being a member of the Higgs sector of the MSSM.

In Fig. 1 we present the tree-level signal cross sections as a function of $M_{H^{ \pm}}$for the LHC and Tevatron colliders. Next in Fig. 2 a rich variety of SUSY effects is exhibited, as a function of $\tan \beta$, for the various sets of MSSM parameters indicated in Table I. We have parametrized these NLO SUSY effects in terms of a SUSY $K$ factor $K_{\mathrm{SUSY}}=\sigma^{\mathrm{SUSY}} / \sigma^{\text {tree }}$. Apart from the MSSM inputs explicitly shown in Table I, the

TABLE I. Typical sets of relevant SUSY parameters used in the computation of the signal cross section (1) in Fig. 2 (all entries in $\mathrm{GeV}$ ). Here $\mu$ and $M$ are the Higgsino and $\mathrm{SU}(2)$ gaugino mass parameters, $m_{\tilde{g}}$ is the gluino mass, $m_{\tilde{t}_{1}}$ and $m_{\tilde{b}_{1}}$ are the lightest stop and sbottom masses, and $A_{t}, A_{b}$ are the top and bottom quark trilinear SUSY-breaking couplings. The sbottom mixing angle is fixed at $\pm \pi / 4$. The $|\delta \rho|<0.001$ constraint is satisfied. Notation as in Ref. [12].

\begin{tabular}{lccccccc}
\hline \hline & $\mu$ & $M$ & $m_{\tilde{g}}$ & $m_{\tilde{t}_{1}}$ & $m_{\tilde{b}_{1}}$ & $A_{t}$ & $A_{b}$ \\
\hline Set A & -1000 & 200 & 1000 & 1000 & 1000 & 500 & 500 \\
Set B & -200 & 200 & 1000 & 500 & 500 & 500 & 500 \\
Set C & 200 & 200 & 1000 & 500 & 500 & -500 & 500 \\
Set D & 1000 & 200 & 1000 & 1000 & 1000 & -500 & 500 \\
\hline \hline
\end{tabular}


rest of the sfermion masses are taken to be around $1 \mathrm{TeV}$; however, we have checked that their concrete values do not affect the results of the present study. The parameter sets $\mathrm{A}-\mathrm{D}$ in Table I represent typical MSSM inputs giving maximum (A and $\mathrm{D})$ and moderate (B and $\mathrm{C}$ ) corrections, of positive $(A$ and $B$ ) and negative $(C$ and $D)$ sign. With this choice of parameters some degree of splitting appears between the third generation squarks. But in all cases these splittings preserve the stringent bounds on the $\rho$ parameter. From the values of $K_{\text {SUSY }}$ for some of these parameter sets it is clear that the MSSM effects can be extraordinarily important in the large $\tan \beta$ region. Indeed, in the relevant cases $K_{\text {SUSY }}$ can be as big or even larger than the maximal QCD $K$ factor expected. We remark that in all parameter sets of Table I we have chosen the situation $\mu A_{t}<0$, which is favored by the data on radiative $B$-meson decays $(b \rightarrow s \gamma)$ [19]. But of the two possibilities, $\mu<0, A_{t}>0$ and $\mu>0, A_{t}<0$, the first one leads to large SUSY $K$ factors above 3 while the second one produces a relatively moderate decrease of the cross section.

The leading corrections are easily identified. They come from the $H^{+} \bar{t} b$ (or $H^{-} t \bar{b}$ ) vertex and can be described through an effective Lagrangian approach [13]

$$
\mathcal{L}=\frac{g V_{t b}}{\sqrt{2} M_{W}} \frac{\bar{m}_{b} \tan \beta}{1+\Delta m_{b}} H^{+} \bar{t}_{L} b_{R}+\text { H.c. }
$$

where $\bar{m}_{b}$ is the running bottom mass in the modified minimal subtraction scheme $(\overline{\mathrm{MS}})$. The previous formula allows one to treat the leading SUSY Yukawa coupling effects correctly resummed to all orders [13]. The analytic form of the strong (SUSY-QCD) and electroweak (SUSY-EW) corrections $\Delta m_{b}$ in the MSSM is given in Ref. [13]. Although $\Delta m_{b}$ is the only correction that contributes at order $(\alpha / 4 \pi) \tan \beta$ ( $\alpha=\alpha_{S}, \alpha_{W}$ ) and thus dominates for large $\tan \beta$, we have also included off-shell SUSY-QCD and SUSY-EW corrections to the $H^{ \pm} t b$ vertex and to the fermion propagators. We have made extensive use of the COMPHEP package for the algebraic and numerical calculations [20]. Despite the fact that COMPHEP is only able (in principle) to deal with treelevel calculations, with the help of Eq. (2) we have managed to add the MSSM corrections to the $H^{ \pm} t b$ vertex and fermion propagators and we have assessed the relevance of the off-shell contributions. To this end we have evaluated the full set of one-loop MSSM diagrams for the relevant $H^{+} t b$ vertex. Notice that the SM electroweak radiative corrections from the gauge sector are much smaller since they are neither enhanced by Yukawa couplings nor by $\tan \beta$. The same set of diagrams was considered in detail in Ref. [12] for the case where all external particles are on shell. In the present instance, however, at least one of the quarks in that vertex is off shell. Therefore, we can use the same bunch of diagrams as in the on-shell case but we have to account for the offshell external lines, which is a nontrivial task. We have studied this issue in detail by expanding the off-shell propagators. First, we have modified COMPHEP's Feynman rules to allow for the most general off-shell $H^{+} \bar{t} b$ vertex; then we let COMPHEP reckon the squared matrix elements and dumped
TABLE II. (a) The main background processes to the signal (1) at the Tevatron (2nd column) and the LHC (3rd column) under the cuts explained in the text. The various contributions are shown together with that of the subtraction term [10]; (b) background from $p p \rightarrow t \bar{t} q g$ when the light quark or gluon are misidentified as a $b$ jet.

(a)

\begin{tabular}{|c|c|c|c|}
\hline & \multirow{4}{*}{$\begin{array}{c}\sigma(q q \rightarrow t \bar{t} b \bar{b}) \\
\sigma(g g \rightarrow t \bar{t} b \bar{b}) \\
\sigma(g b \rightarrow t \bar{t} b) \\
\text { Subtr. term }\end{array}$} & $6.62 \mathrm{fb}$ & $0.266 \mathrm{pb}$ \\
\hline & & $0.676 \mathrm{fb}$ & $6.00 \mathrm{pb}$ \\
\hline & & $1.22 \mathrm{fb}$ & $4.33 \mathrm{pb}$ \\
\hline & & $0.72 \mathrm{fb}$ & $2.1 \mathrm{pb}$ \\
\hline \multirow[t]{3}{*}{ b) } & $\sigma(q \bar{q} \rightarrow g \bar{t} \bar{t})$ & $1890 \mathrm{fb}$ & $21 \mathrm{pb}$ \\
\hline & $\sigma(g q \rightarrow q t \bar{t})$ & $193 \mathrm{fb}$ & $122 \mathrm{pb}$ \\
\hline & $\sigma(g g \rightarrow g \bar{t} \bar{t})$ & $262 \mathrm{fb}$ & $371 \mathrm{pb}$ \\
\hline
\end{tabular}

the result into REDUCE code. The subsequent numerical computation of the one-loop integrals has been done using the package LOOPTOOLS [21]. At this point, we have inserted expressions for the coefficients of the off-shell $H^{+} \bar{t} b$ vertex that include the one-loop off-shell supersymmetric corrections to the vertex itself and to the off-shell fermion propagators and fermionic external lines. Only half the renormalization of an internal fermion line has to be included, the other half being associated to the $g q q$ vertex. This procedure has allowed us to estimate the relative size of the off-shell effects in the signal cross section, which never exceeds the few percent level. The upshot is that the approximation of neglecting vertex and propagator corrections in the cross section, which may be called "improved Born" approximation, is really justified in the relevant region of parameter space.

As for the signal versus background study we have focused on the $t \bar{t} b \bar{b}$ signature corresponding to the $H^{+} \rightarrow t \bar{b}$ decay channel, which has the biggest branching ratio at high $\tan \beta$. Then we have concentrated on the triple- $b$ tagging case, which gives the best possibility to measure the signal cross section (1). This is a crucial point, especially for the Tevatron, where the production rate is too small to give any viable signal in the case of four- $b$ tagging. As regards the LHC, a triple- $b$ tagging study allows the signal cross-section to be measured more precisely, even though the signal/ background ratio can be better for the four- $b$ tagging case. We notice that the background processes in Table II are insensitive to the leading type of MSSM quantum effects that contribute to Eq. (1). To perform a realistic signal and background event simulation we complied with the following procedure. The (tree-level) matrix elements for the signal and background processes have been calculated using the COMPHEP package [20]. The next step was the parton-level event simulation, also with the help of COMPHEP. Then we automatically linked the parton-level events from COMPHEP to the PYTHIA 6.1 Monte Carlo generator and the COMPHEPPYTHIA interface [22]. Therefore we took into account the effects of the final-state radiation, hadronization, and stringjet fragmentation using PYTHIA tools. The following resolutions were used for the jet and electron energy smearing: $\Delta E^{\text {had }} / E=0.8 / \sqrt{E}$ and $\Delta E^{\text {ele }} / E=0.2 / \sqrt{E}$. In our analysis we 
used the cone algorithm for the jet reconstruction with a cone size $\Delta R=\sqrt{\Delta \varphi^{2}+\Delta \eta^{2}}=0.7$. The choice of this jet-cone value is related to the crucial role of the final-state radiation (FSR), which strongly smears the shape of the reconstructed charged Higgs boson mass. We have checked that the value of 0.7 minimizes the FSR effects. Now, in order to decide whether a charged Higgs cross-section leads to a detectable signal, we have to compute the background rate. Since the mistagging probability of light quark and gluon jets is expected to be $\leqslant 1 \%$ [23], the only significant backgrounds leading to the same $t \bar{t} b \bar{b}$ signature are those shown in Table II along with their respective cross sections. For the $t \bar{t} b \bar{b}$ and $t \bar{t} q g$ processes we have applied the jet separation cut $\Delta_{R}^{j j}>0.5\left(\Delta_{R}=\sqrt{\Delta \theta^{2}+\Delta \phi^{2}}\right)$ and the initial cut $p_{T}^{j}$ $>10 \mathrm{GeV}\left(p_{T}^{j}>20 \mathrm{GeV}\right)$ at the Tevatron (LHC). For the $t \bar{t} j$ process the initial cut $p_{T}^{j}>10 \mathrm{GeV}\left(p_{T}^{j}>20 \mathrm{GeV}\right)$ was applied at the Tevatron (LHC). To obtain a realistic description of the $b$-tagging efficiency as a function of $b$-quark transverse momenta, for the Tevatron we use the projected $b$-tagging efficiency of the upgraded $\mathrm{D} \emptyset$ detector [9] while for the LHC we parametrize numerical results from the CMS Collaboration [23]. Efficiencies for both parametrizations are about $60 \%$ at the $p_{T}^{b}$ saturation value of $\sim 100 \mathrm{GeV}$. We assume that $b$ jets can be tagged only for pseudorapidity $\left|\eta_{b}\right| \leqslant 2$ by both Tevatron and LHC experiments. Furthermore, we have optimized the reconstruction procedure, the $p_{T}$ cut on the leading $b$ jet $\left(p_{T}^{b}>\left[M_{H^{+}} / 5-15\right] \mathrm{GeV}\right)$ and window cut on the $t b$-invariant mass around the selected values of $M_{H^{+}}\left(\left|m_{t b}-M_{H^{+}}\right|<5 \sqrt{M_{H^{+}}}\right)$to achieve the maximal significance of the cross-section signal $\sigma_{S}$ (1) versus the background $\sigma_{S} / \sqrt{B}$. The typical efficiency at the Tevatron is $5-6 \%$ while for LHC it goes down to $1-2 \%$. These values include the triple $b$-tagging branchings of $W$-bosons decays (leptonic and hadronic decay modes) and the efficiency of the kinematical cuts and reconstruction of the $t \bar{t} b \bar{b}$ signature. As an example, Fig. 3 shows the reconstructed $t b$ invariant-mass distribution for signal and background events at the LHC.

In Figs. 4 and 5 we present the discovery and exclusion limits for $H^{ \pm}$at the Tevatron and the LHC, respectively, at high $\tan \beta$ : They show the signal cross-section $\sigma_{S}$ and the cross sections which would lead to the $5 \sigma, 3 \sigma$, and $1.98 \sigma$ (95\% C.L.) significance. From the intersections of the last three with $\sigma_{S}$ we infer the $H^{ \pm}$-mass ranges which can be discovered or excluded. The tree-level case is also shown, and it is seen to be too small at the Tevatron to place any limit. But when one includes the MSSM quantum effects, say the moderate input set B from Table I, the situation changes. For example, for $M_{H^{ \pm}}=215 \mathrm{GeV}$ at the Tevatron one would expect (with $K=1$ ) seven signal and about six background reconstructed events at $L=25 \mathrm{fb}^{-1}$. At the LHC with $M_{H^{ \pm}}=500 \mathrm{GeV}$ we have 1200 and 3800 signal and background events, respectively, at $L=100 \mathrm{fb}^{-1}$. A canonical $5 \sigma$ discovery limit around $800 \mathrm{GeV}$ can be obtained at the LHC for the MSSM charged Higgs, or else an exclusion limit at $95 \%$ C.L. up to at least $1.2 \mathrm{TeV}$. For the Tevatron we can only place a $95 \%$ C.L. exclusion limit in the mass

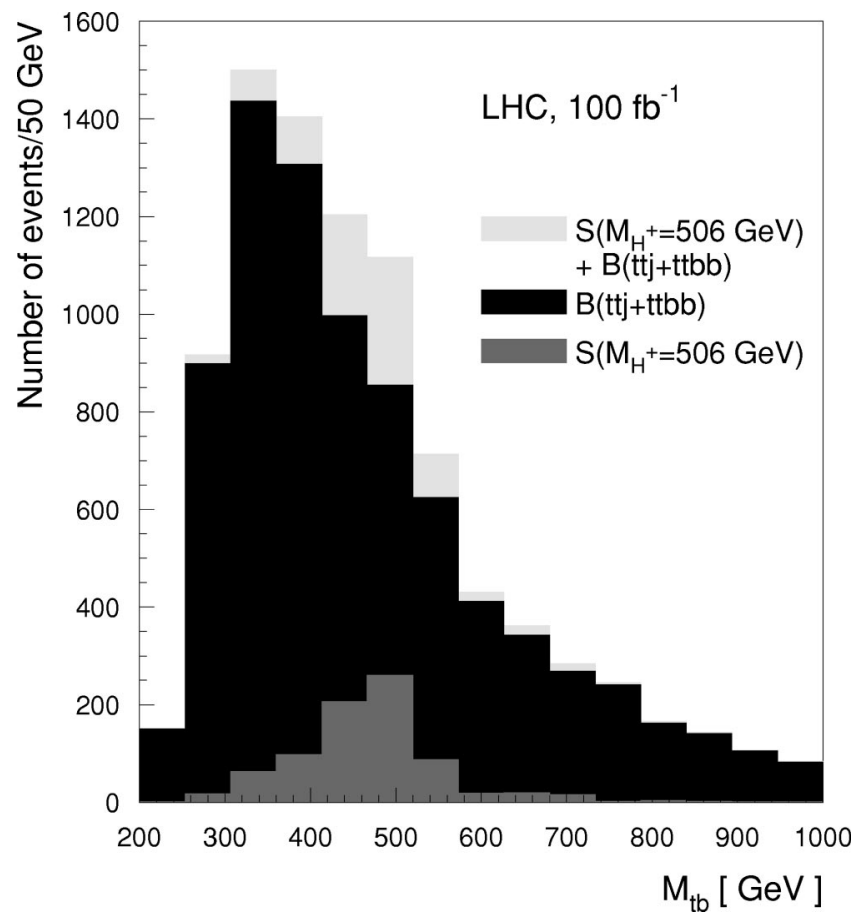

FIG. 3. Reconstructed $t b$ invariant-mass distribution for signal, background and signal plus background events, for $M_{H^{ \pm}}$ $=500 \mathrm{GeV}$, at the LHC.

range 200-250 GeV. However, if the QCD $K$ factor lies around the previous estimate, say at $K=1.5$, this would open the exciting opportunity to observe the charged Higgs already at the Tevatron with a $3 \sigma$ significance in the mass range $220-250 \mathrm{GeV}$. We stress that this chance would not be possible with the QCD corrections $K \sim 1.5$ alone, but only after folding them with MSSM quantum effects of (at least) the same order. Finally, we notice that $b$-tagging efficiency could be increased up to $\sim 70 \%$ with the use of the $3 \mathrm{D}$ vertexing algorithms [5]. For triple $b$ tagging, this would

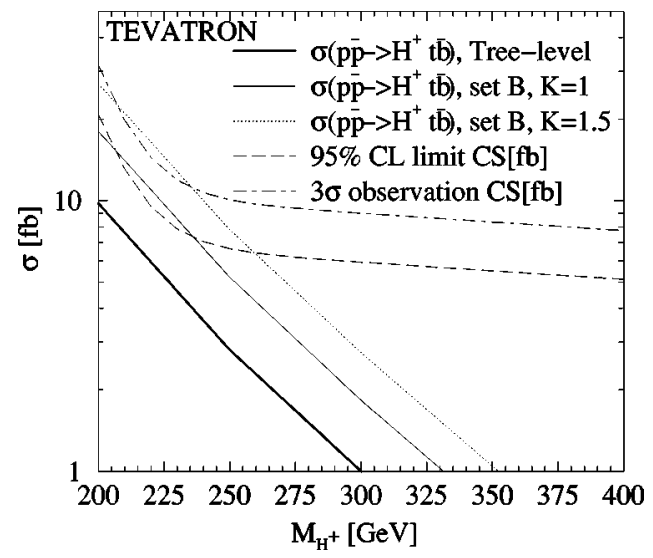

FIG. 4. Discovery and exclusion limits for the charged Higgs boson at the Tevatron, for $\tan \beta=50$. Shown are the total cross sections for (i) the signal (1) at the tree-level, (ii) the SUSY corrected signal for the input set $\mathrm{B}$ in Table I including QCD factors $K=1$ and $K=1.5$, (iii) the $3 \sigma$ discovery limit, (iv) the 95\% C.L. exclusion limit. 


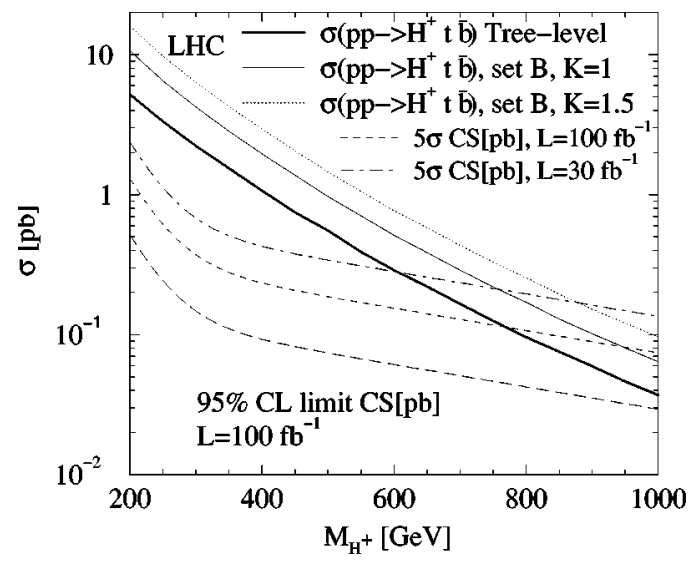

FIG. 5. As in Fig. 4 but for the LHC. The $5 \sigma$ discovery limits are shown for the two integrated luminosities $L=\int \mathcal{L} d t=30 \mathrm{fb}^{-1}$ and $100 \mathrm{fb}^{-1}$, and the $95 \%$ C.L. exclusion limit is for $L$ $=100 \mathrm{fb}^{-1}$.

augment the signal by at least a factor of 2 , and so the discovery and the $95 \%$ exclusion limits would be extended accordingly. Needless to say, in case of maximal SUSY enhancement (cf. set A) the exclusion/observation mass range for the charged Higgs boson would be further enlarged.

In summary, we have assessed the possibility of seeing a SUSY charged Higgs boson at the Tevatron and the LHC through the mechanisms (1). Our study of the quantum corrections within the MSSM has shown that at high $\tan \beta$ they are dominated by exceptionally important effects that can be absorbed into an effective $H^{ \pm} t b$ vertex, and therefore in practice they can be treated as an "improved Born approximation." From a detailed signal versus background study we have shown that the prospects for the signal discovery are viable for the Tevatron (if $M_{H^{ \pm}} \lesssim 250 \mathrm{GeV}$ ) and promising for the LHC (if $M_{H^{ \pm}} \leq 1.5 \mathrm{TeV}$ ). In deriving these limits we have demonstrated that the quantum machinery from the MSSM can play a crucial role to increase the signal. In large portions of the parameter space the MSSM corrections could show up as a smoking gun over the tree-level cross section (after subtracting the conventional QCD effects, once they will be known). Since both the processes (1) and the SUSY effects are only relevant at high $\tan \beta$, the sole presence of the signal could be a hallmark of $\tan \beta \gg 1$ and of the underlying MSSM dynamics.

The work of D.G. and J.G. has been supported by the E.U. Marie Curie program, and that of J.S. by CICYT. J.S. is thankful to M. Spira for interesting discussions on the role played by the QCD corrections. A.B. thanks J. Owens, N. Kidonakis, and L. Reina for stimulating discussions and acknowledges the support of U.S. DOE.
[1] ALEPH Collaboration, R. Barate et al., Phys. Lett. B 495, 1 (2000); L3 Collaboration, M. Acciarri et al., ibid. 495, 18 (2000).

[2] J.F. Gunion, H.E. Haber, G.L. Kane, and S. Dawson, The Higgs Hunters' Guide (Addison-Wesley, Menlo-Park, 1990).

[3] J.F. Gunion, Phys. Lett. B 322, 125 (1994); V. Barger, R.J. Phillips, and D.P. Roy, ibid. 324, 236 (1994).

[4] Z. Kunszt, Nucl. Phys. B247, 339 (1984); J.F. Gunion, H.E. Haber, F.E. Paige, W. Tung, and S.S. Willenbrock, ibid. B294, 621 (1987); D.A. Dicus and S. Willenbrock, Phys. Rev. D 39, 751 (1989); W.J. Marciano and F.E. Paige, Phys. Rev. Lett. 66, 2433 (1991).

[5] J. Goldstein, C.S. Hill, J. Incandela, S. Parke, D. Rainwater, and D. Stuart, Phys. Rev. Lett. 86, 1694 (2001).

[6] M. Carena, S. Mrenna, and C.E.M. Wagner, Phys. Rev. D 60, 075010 (1999); M. Spira, in [9] and in Quantum Effects in the MSSM, edited by J. Solà (World Scientific, Singapore, 1998), p. 125 .

[7] F. Borzumati, J. Kneur, and N. Polonsky, Phys. Rev. D 60, 115011 (1999); D.J. Miller, S. Moretti, D.P. Roy, and W.J. Stirling, ibid. 61, 055011 (2000); L.G. Jin, C.S. Li, R.J. Oakes, and S.H. Zhu, Eur. Phys. J. C 14, 91 (2000), and references therein.

[8] J.A. Coarasa, J. Guasch, and J. Solà, hep-ph/9909397, contributed to Ref. [9].

[9] M. Carena et al., "Report of the Tevatron Higgs working group of the Tevatron Run 2 SUSY/Higgs Workshop," hep-ph/0010338.
[10] See, e.g., D. Dicus, T. Stelzer, Z. Sullivan, and S. Willenbrock, Phys. Rev. D 59, 094016 (1999), and references therein.

[11] A. Belyaev and E. Boos, Phys. Rev. D 63, 034012 (2001).

[12] J.A. Coarasa, D. Garcia, J. Guasch, R.A. Jiménez, and J. Solà, Eur. Phys. J. C 2, 373 (1998); Phys. Lett. B 425, 329 (1998), and references therein.

[13] M. Carena, D. Garcia, U. Nierste, and C.E.M. Wagner, Nucl. Phys. B577, 88 (2000).

[14] W. Beenakker, S. Dittmaier, M. Krämer, R. Plümper, M. Spira, and P.M. Zerwas, Phys. Rev. Lett. 87, 201805 (2001); L. Reina and S. Dawson, ibid. 87, 201804 (2001); L. Reina, S. Dawson, and D. Wackeroth, Phys. Rev. D (to be published), hep-ph/0109066.

[15] S. Dawson and L. Reina, Phys. Rev. D 57, 5851 (1998).

[16] P. Nason, S. Dawson, and R.K. Ellis, Nucl. Phys. B303, 607 (1988); W. Beenakker, H. Kuijf, W.L. van Neerven, and J. Smith, Phys. Rev. D 40, 54 (1989).

[17] S. Dittmaier, M. Krämer, Y. Liao, M. Spira, and P.M. Zerwas, Phys. Lett. B 478, 247 (2000).

[18] M. Spira (private communication).

[19] Particle Data Group, D.E. Groom et al., Eur. Phys. J. C 15, 1 (2000).

[20] A. Pukhov et al., hep-ph/9908288.

[21] T. Hahn and M. Pérez-Victoria, Comput. Phys. Commun. 118, 153 (1999).

[22] T. Sjostrand, Comput. Phys. Commun. 82, 74 (1994); A.S. Belyaev et al., hep-ph/0101232.

[23] CMS Collaboration, S. Abdullin et al., hep-ph/9806366. 